\title{
Helicobacter pylori potentiates epithelial:mesenchymal transition in gastric cancer: links to soluble HB-EGF, gastrin and matrix metalloproteinase-7
}

\author{
Yinfei Yin, ${ }^{1}$ Anna M Grabowska, ${ }^{1}$ Philip A Clarke, ${ }^{1}$ Elisabeth Whelband, ${ }^{1}$ \\ Karen Robinson, ${ }^{2}$ Richard H Argent, ${ }^{1}$ Amanda Tobias, ${ }^{1}$ Rajendra Kumari, ${ }^{1}$ \\ John C Atherton, ${ }^{3}$ Susan A Watson ${ }^{1}$
}

${ }^{1}$ Division of Pre-Clinical

Oncology, University of Nottingham, Nottingham, UK

${ }^{2}$ Division of Medicine, University of Nottingham, Nottingham, UK ${ }^{3}$ Nottingham Digestive Diseases Centre, School of Clinical Sciences, University of Nottingham, Nottingham, UK

\section{Correspondence to} Susan A Watson, Division of Pre-Clinical Oncology, D Floor, West Block, Queen's Medical Centre, University Hospital, University of Nottingham, Nottingham NG7 2UH, UK; sue.watson@nottingham.ac.uk

Revised 8 February 2010 Accepted 13 March 2010 28 June 2010 Published Online First

\begin{abstract}
Background and aims Helicobacter pylori (H pylori) infection is a major risk factor in the development of distal gastric adenocarcinoma. Development of the invasive phenotype is associated with the phenomenon of epithelial:mesenchymal transition (EMT). Soluble heparin-binding epidermal growth factor (HB-EGF) has been implicated in this process. A study was undertaken to investigate the possibility that matrix metalloproteinase (MMP)-7 is upregulated in $\mathrm{H}$ pylori infection as a result of hypergastrinaemia, which may enhance shedding of HB-EGF and contribute towards EMT in gastric adenocarcinoma cell lines.

Methods Three gastric epithelial cell lines (AGS, MGLVA1 and ST16) were co-cultured with the pathogenic $H$ pylori strain 60190 and non-pathogenic strain Tx30a in an in vitro infection model. Gene expression was quantified by real-time PCR, HB-EGF shedding by ELISA and protein expression by immunofluorescence or immunohistochemistry. The INSGAS mouse, a transgenic mouse model of gastric carcinogenesis which overexpresses amidated gastrin, was used to investigate the in vivo relationship between HB-EGF, MMP-7, gastrin and EMT.
\end{abstract}

Results The pathogenic strain of $H$ pylori significantly upregulated EMT-associated genes Snail, Slug and vimentin in all three gastric cell lines to a greater degree than the non-pathogenic strain. Pathogenic $\mathrm{H}$ pylori also upregulated HB-EGF shedding, a factor implicated in EMT, which was partially dependent on both gastrin and MMP-7 expression. Gastrin and MMP-7 siRNAs and MMP-7 neutralising antibody significantly reduced upregulation of HB-EGF shedding in $\mathrm{H}$ pylori infected gastric cell lines and reduced EMT gene expression. The effect of $H$ pylori on EMT was also reversed by gastrin siRNA. Neutralisation of gastrin in the INS-GAS mouse model reduced expression of MMP-7, HB-EGF and key EMT proteins

Conclusion The upregulation of MMP-7 by pathogenic $H$ pylori is partially dependent on gastrin and may have a role in the development of gastric cancer, potentially through EMT, by indirectly increasing levels of soluble HB-EGF.

\section{INTRODUCTION}

This paper is freely available online under the BMJ Journals unlocked scheme, see http:// gut.bmj.com/site/about/ unlocked.xhtml
Helicobacter pylori infection is the greatest risk factor for gastric cancer and $H$ pylori infects approximately $40 \%$ of the population in developed coun-

\section{Significance of this study}

What is already known about this subject?

- $H$ pylori infection is the greatest risk factor for gastric cancer.

- $H$ pylori infection up-regulates HB-EGF, MMP7 and gastrin expression.

- HB-EGF and MMP-7 have been linked to EMT.

What are the new findings?

- EMT gene expression is up-regulated in gastric epithelial cells infected with a pathogenic strain of $H$ pylori.

- MMP-7 plays a role in the up-regulation of soluble HB-EGF shedding and EMT induced by $H$ pylori infection.

- Gastrin is involved in $H$ pylori-induced MMP-7 and EMT up-regulation.

- Neutralisation of gastrin in an INS-GAS mouse in vivo carcinogenesis model leads to reduced proliferation and MMP-7, Slug and HB-EGF expression.

How might it impact on clinical practice in the foreseeable future?

- HB-EGF, gastrin and MMP-7 may serve as potential targets for the development of novel therapeutics for patients with $\mathrm{H}$ pylori-induced gastric cancer.

tries by the age of $50 .{ }^{1}$ However, only about $0.4 \%$ of the infected population develop gastric cancer. ${ }^{2}$ Many factors affect the final outcome of $H$ pylori infection. These include the $H$ pylori cag pathogenicity island (cagPaI), which is a $35-40 \mathrm{~kb}$ genetic element that encodes a type IV secretion system and is strongly associated with gastric malignant progression. ${ }^{3} 4$

Heparin-binding epidermal growth factor (HBEGF) is a member of the epidermal growth factor (EGF) family and functions by activation of the EGF receptor and other ErbB receptors. ${ }^{5}$ Both HB-EGF gene expression and protein shedding are increased in $H$ pylori infection. ${ }^{6}$ Recent data suggest that soluble HB-EGF has a role in epithelial: mesenchymal transition (EMT) via upregulation of members of the EMT transcriptome including Slug.7 ${ }^{8}$ Interestingly, $H$ pylori cagA was shown to induce an EMT-like effect when expressed in epithelial cells, although the mechanism was not clarified. ${ }^{9}$ HB-EGF enhances cell motility as well as 
contributing to cancer invasion and metastasis. ${ }^{10} 11$ Conversely, membrane-bound HB-EGF appears to be responsible for upregulation of E-cadherin, minimising the potential of EMT. ${ }^{12}$

HB-EGF is initially synthesised as a transmembrane precursor protein which is cleaved at the cell surface to yield a mature soluble form of HB-EGF. ${ }^{13}$ The matrix metalloproteinase (MMP) family is believed to play a role in HB-EGF ectodomain shedding, which is essential for biological activity of the growth factor. ${ }^{13}$ Epithelial-associated MMP-7 is a downstream transcriptional target of $\beta$-catenin following E-cadherin deregulation and has previously been linked to EMT and upregulated in $H$ pylori infection. ${ }^{14-16}$

A common denominator that has been linked to $H$ pyloriinduced MMP-7 and HB-EGF expression and shedding is gastrin, which is upregulated along with its receptor, the gastrin/CCK-2 receptor, in gastric epithelial cells by $H$ pylori at both gene and protein levels. ${ }^{6}{ }^{17}$ Furthermore, gastrin can lead to overexpression of various MMPs including MMP-7 in conditions of hypergastrinaemia which may activate EMT and contribute to stromal infiltration. $^{18-20}$

Based on these findings, we hypothesised that the ability of $H$ pylori infection to induce EMT in the gastric malignant environment was via gastrin-induced expression of MMP-7 leading to increased levels of soluble HB-EGF.

The described studies attempt to dissect out the inter-relationships between gastrin, HB-EGF and MMP-7 in terms of EMT induction on the background of $H$ pylori-infected malignant gastric epithelial cells.

\section{MATERIALS AND METHODS \\ Cell culture}

Three human gastric adenocarcinoma cell lines, AGS (European Collection of Animal Cell Cultures, Wiltshire, UK), MGLVA1 and ST16 (Division of Pre-Clinical Oncology, University of Nottingham, Nottingham, UK), were used in this study. ${ }^{21}$ Cells were cultured in RPMI 1640 (Sigma-Aldrich, Dorset, UK) supplemented with $10 \%(\mathrm{v} / \mathrm{v})$ heat-inactivated foetal bovine serum (Sigma) and $2 \mathrm{mM}$ L-glutamine (Sigma) in an atmosphere of $5 \% \mathrm{CO}_{2}$ at $37^{\circ} \mathrm{C}$.

\section{siRNA transfection}

Cells were transfected with specifically designed small interfering RNAs (siRNAs) to knock down either gastrin (5'-UCCAUCCAUAGGCUUCUUCUU-3') or MMP-7 (5'UUCAUGAGUUGCAGCAUACUU-3'). ${ }^{22}$ A universal negative control siRNA was used as a negative control (Eurogentec, Southampton, Hampshire, UK). Transfection was carried out using siPORT Amine siRNA transfection reagent (Ambion, Warrington, UK) following the manufacturer's instructions as described previously. ${ }^{23}$ After transfection, cells were cultured in a normal cell culture environment for $48 \mathrm{~h}$ before further experimentation

\section{Bacterial co-culture}

$H$ pylori strains 60190 (American Type Culture Collection (ATCC) 49503) and Tx30a (ATCC 51932) were used in this study. Strain 60190 expresses an intact and functional cagPaI and possesses an s1/m1 vacA toxin while strain Tx30a expresses $\mathrm{s} 2 \mathrm{~m} 2$ vacA toxin but does not possess the cagPaI. The bacteria were routinely cultured on $5 \%$ horse blood agar plates (Oxoid Ltd, Basingstoke, UK) in humidified incubators which provide an atmosphere of $5 \% \mathrm{CO}_{2}$ at $37^{\circ} \mathrm{C}$.
Gastric epithelial cells were trypsinised, resuspended in normal growth medium and seeded into 6 or 24 well plates. Three duplicate wells were prepared for each experimental condition. When the cells reached $70 \%$ confluence they were serum-starved for $24 \mathrm{~h}$ prior to addition of $H$ pylori at a multiplicity of infection of 200. Cells were co-cultured with the bacteria for $6 \mathrm{~h}$ before either RNA extraction or protein measurements were performed.

A MMP-7 neutralising antibody (R\&D, Abingdon, UK) was used at $2 \mu \mathrm{g} / \mathrm{ml}$ to inhibit MMP-7 activity in the co-culture model. This antibody has the ability to neutralise MMP-7 activity according to manufacturer's description.

\section{RNA extraction, reverse transcription and real-time PCR}

After co-culture, medium was removed and the cells were washed with phosphate buffered saline (PBS). The cells were then lysed in $1 \mathrm{ml}$ TRI-reagent and RNA extracted following the manufacturer's recommendations (Sigma). Total RNA was incubated with random primers and SuperScript II (Invitrogen, Paisley, UK) at $25^{\circ} \mathrm{C}$ for $10 \mathrm{~min}, 42^{\circ} \mathrm{C}$ for $1 \mathrm{~h}$ and $95^{\circ} \mathrm{C}$ for $10 \mathrm{~min}$ in reverse transcription reaction buffer to make cDNA.

PCRs were carried out in 96-well optical reaction plates using $1 \mu \mathrm{l} \mathrm{cDNA}$ in a $25 \mu \mathrm{l}$ reaction mix consisting of $1 \times$ reaction buffer, 1:2000 SYBR Green, $\mathrm{MgCl}_{2}$, deoxynucleotide triphosphate mix, primers (table 1) and HotGoldStar Taq (all from Eurogentec). The samples were run on a GeneAmp 7500 Sequence Detector Real-time PCR machine (Applied Biosystems, Foster City, California, USA) using the following program: $50^{\circ} \mathrm{C}$ for $2 \mathrm{~min}, 95^{\circ} \mathrm{C}$ for $10 \mathrm{~min}$, then 40 cycles of $95^{\circ} \mathrm{C}$ for $15 \mathrm{~s}$ and $60^{\circ} \mathrm{C}$ for $1 \mathrm{~min}$. The level of the test gene was compared with the housekeeping gene hypoxanthine phosphoribosyltransferase (HPRT). The results are presented as relative gene expression compared with HPRT using $2^{-\Delta \Delta C t} \cdot{ }^{24}$

\section{ELISA for secreted HB-EGF detection}

Cells were washed with $2 \mathrm{M} \mathrm{NaCl}$ and the eluate was collected and clarified through a $2 \mu \mathrm{m}$ filter. Cells were seeded at a concentration of $3 \times 10^{5} /$ well at the beginning of the experiments to normalise the protein level.

Ninety-six-well plates were coated by adding the capture antibody (R\&D Systems) at $100 \mu \mathrm{l} /$ well and incubating overnight at room temperature. The plates were washed four times with PBS-Tween 20 (PBS-T) after each step, then blocked with $1 \%$ bovine serum albumin (BSA) (Sigma) in PBS for $2 \mathrm{~h}$ at room

Table 1 Primer sequences

\begin{tabular}{lll}
\hline Gene & & Sequence $\mathbf{5}^{\prime}-\mathbf{3}^{\prime}$ \\
\hline Gastrin & Forward & CCACACCTCGTGGCAGAC \\
& Reverse & TCCATCCATCCATAGGCTTC \\
HPRT & Forward & GACCAGTCAACAGGGGACAT \\
& Reverse & CGACCTTGACCATCTTTGGA \\
MMP-7 & Forward & GATGGTAGCAGTCTAGGGATTAACTTC \\
& Reverse & GGAATGTCCCATACCCAAAGAA \\
HB-EGF & Forward & CTCTTCT GGCTGCAGTTCTC \\
& Reverse & AGCTGGTCCGTGGATACAGT \\
Snail & Forward & CCCCAATCGGAAGCTAACT \\
& Reverse & GGTCGTAGGGCTG CTGGAA \\
Slug & Forward & CTGCGGCAAGGCGTT \\
& Reverse & GCAGTGAGGGCAAGAAAAGG \\
Vimentin & Forward & AAAACACCCTGCAATCTTTCAGA \\
& Reverse & CACTTTGCGTTCAAGGTCAAGAC
\end{tabular}

HB-EGF, heparin-binding epidermal growth factor; HPRT, hypoxanthine phosphoribosyltransferase; MMP, matrix metalloproteinase. 
temperature. $100 \mu \mathrm{l} /$ well of sample or serial diluted recombinant HB-EGF (R\&D) was added and the plates incubated at room temperature for $2 \mathrm{~h}$. $100 \mathrm{ng} / \mathrm{ml}$ detection antibody (R\&D) was added and the plates incubated for a further $2 \mathrm{~h}$. Binding of the antibody was detected by the addition of horseradish peroxidase-conjugated streptavidin (Dako, Ely, UK) and 3,3',5,5'tetramethylbenzidine substrate (Sigma). The reaction was stopped with $0.5 \mathrm{M} \mathrm{H}_{2} \mathrm{SO}_{4}$ and the absorbance read at $450 \mathrm{~nm}$. As recombinant HB-EGF was added at serial dilutions of known concentration, a standard curve was calculated by use of the graphical package Graphpad Prism. HB-EGF concentration was then determined by referring to the standard curve.

\section{Animal sample collection and fixation}

Insulin-gastrin (INS-GAS) mice are transgenic mice overexpressing amidated gastrin in pancreatic islets that leads to accelerated gastric carcinogenesis. $^{25}$ An INS-GAS mouse develops gastric metaplasia, dysplasia, carcinoma and gastric cancer with vascular invasion at 20 months of age; this model shows a direct link between gastrin and gastric cancer. INS-GAS mice were given a course of either $250 \mathrm{mg} / \mathrm{kg}$ gastrin immunogen G17DT (amino terminal portion of gastrin-17 fragment linked to carrier protein diphtheria toxoid (DT)) or control immunogen DT at 0, 1 and 3 weeks and then at 3-weekly intervals thereafter. At the study end point, mice were administered bromodeoxyuridine (BrdU) $1 \mathrm{~h}$ before termination. ${ }^{26}$

The mice were then anaesthetised and killed by cervical dislocation and the stomachs were dissected out and washed in PBS. For formalin fixation, samples were preserved in formal calcium (Sigma) for $48 \mathrm{~h}$ before being processed in a carousel processor (TP1020, Leica). For frozen fixation, samples were immersed in Optimum Cutting Temperature compound (Bayer PLC, Berkshire, UK) and snap frozen in liquid nitrogen. Samples were sectioned at $0.4 \mu \mathrm{m}$ and mounted onto poly-lysine coated slides (Fisher Scientific, Leicestershire, UK).

\section{Immunohistochemistry staining}

All staining steps were carried out at room temperature and samples were washed with PBS between steps. Sections were dewaxed in three changes of xylene for $3 \mathrm{~min}$ each and microwave heated to $98^{\circ} \mathrm{C}$ for $15 \mathrm{~min}$ (EMS900, Electron Microscopy Sciences, Hatfield, USA) in citric acid buffer ( $\mathrm{pH}$ 6.0). Sections were then incubated with primary antibodies or commercially prepared universal controls (Dako) for $1 \mathrm{~h}$. The primary antibodies used were rabbit anti-MMP-7 and rabbit anti-HB-EGF (Abcam, Cambridge, UK). Biotinylated swine anti-rabbit (Dako) secondary antibodies were applied for another hour following the removal of the primary antibodies. Staining was developed with avidin-biotinylated horseradish peroxidase complex (Dako) and diaminobenzidine (Dako) following the manufacturer's instructions.

\section{Immunofluorescence staining}

For tissue samples, frozen sections were brought to room temperature, air dried for $30 \mathrm{~min}$ and fixed in $4 \%$ paraformaldehyde (Sigma) for $10 \mathrm{~min}$.

Cells were washed with ice-cold PBS, fixed with $4 \%$ paraformaldehyde (Sigma) for $10 \mathrm{~min}$ before being cytospun at $1500 \mathrm{rpm}$ for $5 \mathrm{~min}$ (Thermo Scientific Cytospin 4 Cytocentrifuge) onto poly-lysine slides.

Slides were washed twice in PBS for 5 min and blocked in 3\% BSA, then $1 \%$ glycine and $10 \%$ normal serum in PBS for 60 min at room temperature. The blocking mixture was aspirated before slides were incubated with primary antibody at $4^{\circ} \mathrm{C}$ overnight. The slides were washed three times with PBS for $5 \mathrm{~min}$ each and secondary antibody applied for $30 \mathrm{~min}$. The slides were then washed again before counterstaining with Hoechst and coverslipped with CitiFluor AF2 antifade mounting agent (Electron Microscopy Sciences). All primary antibodies were purchased from Abcam and all secondary antibodies were from Invitrogen.

\section{Statistical methods}

The results were analysed using a Student t test or one-way ANOVA. The statistical analysis software used was SPSS Version 14.0 .

\section{RESULTS}

\section{EMT gene expression is upregulated in gastric epithelial cells} infected with a pathogenic strain of $\boldsymbol{H}$ pylori

Snail and Slug gene expression was upregulated in all three human gastric epithelial cell lines by the pathogenic $H$ pylori strain 60190 (Snail: AGS $p<0.001$, MGLVA1 $p<0.05$, ST16 $p<0.005$; Slug: $p<0.001$; figure $1 \mathrm{a}$ and $b)$. The non-pathogenic $H$ pylori strain TX30a upregulated Snail (AGS, $\mathrm{p}<0.005$; MGLVA1, $\mathrm{p}<0.05$; ST16, $\mathrm{p}<0.02)$ but to a significantly lower level in the AGS and ST16 cell lines $(\mathrm{p}<0.05$; figure 1a). Tx30a also upregulated Slug (AGS, $p<0.001$; MGLVA1, $\mathrm{p}<0.005$; ST16,
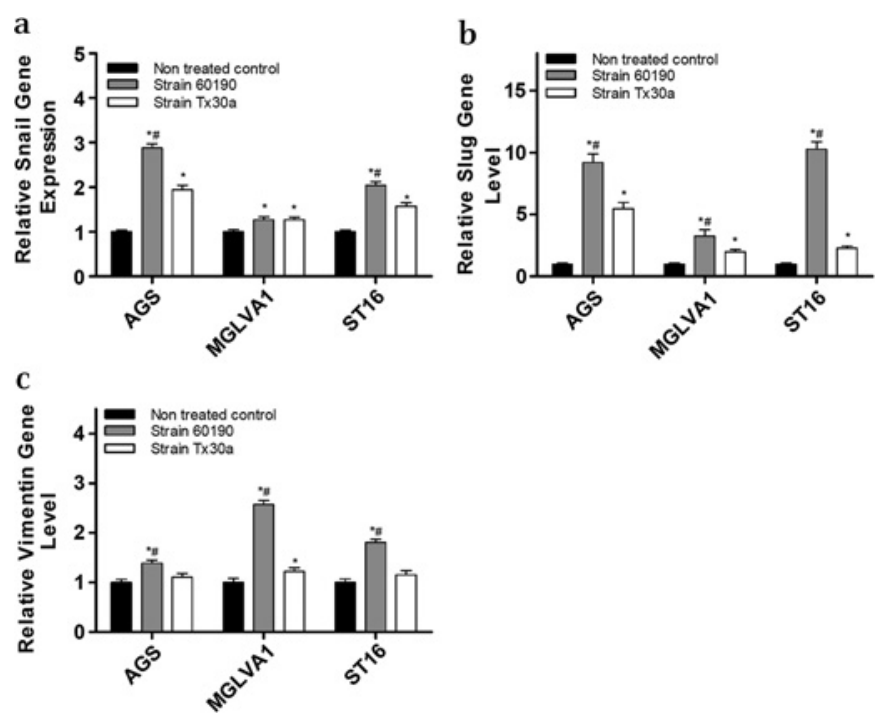

Figure 1 Epithelial:mesenchymal transition gene expression following co-culture of gastric epithelial cells with Helicobacter pylori pathogenic (60190) and non-pathogenic (Tx30a) strains. (a) Snail gene expression: * significantly higher gene expression level compared with untreated control cells (strain 60190 treated: AGS $p<0.001$, MGLVA1 $p<0.05$, ST16 $p<0.005$; strain Tx30a treated: AGS $p<0.005$, MGLVA1 $p<0.05$, ST16 $p<0.02)$; \#significantly higher gene expression level compared with Tx30a treated cells (AGS $p<0.05$, ST16 $p<0.05$ ). (b) Slug gene expression: * ${ }^{*}$ significantly higher gene expression level compared with untreated control cells (strain 60190 treated: $p<0.001$ in all cell lines; strain Tx30a treated: AGS $p<0.001$, MGLVA1 $p<0.005$, ST16 $p<0.005$ ); \#significantly higher gene expression level compared with Tx30a treated cells (AGS $p<0.04$, MGLVA1 $p<0.05$, ST16 $p<0.002$ ).

(c) Vimentin gene expression: * significantly higher gene expression level compared with untreated control cells (strain 60190 treated: AGS $\mathrm{p}<0.05$, MGLVA1 $\mathrm{p}<0.001$, ST16 $\mathrm{p}<0.005$; strain Tx30a treated: MGLVA1 $\mathrm{p}<0.05)$; \# significantly higher gene expression level compared with Tx30a treated cells (MGLVA1 $p<0.04$ ). ( $n=3$ replicates per condition and a representative graph is shown, error bars indicate $95 \%$ confidence). 
$\mathrm{p}<0.005)$ but to a significantly lower level compared with strain 60190 in all three gastric cell lines (AGS, $p<0.04$; MGLVA1, $p<0.05$; ST16, $p<0.002$; figure $1 b$ ). The mesenchymal gene vimentin was also upregulated in the three gastric lines following infection with strain 60190 (AGS, $p<0.05$; MGLVA1, $\mathrm{p}<0.001$; ST16, $\mathrm{p}<0.005$; figure 1c) but was only upregulated in one of the three gastric lines (MGLVA1, $p<0.05$ ) following infection with strain Tx30a (figure 1c) at a significantly lower level $(p<0.04)$.

\section{Upregulation of HB-EGF soluble protein in gastric epithelial cells infected with a pathogenic strain of $\boldsymbol{H}$ pylori}

It is known that soluble HB-EGF increases EMT gene expression and expression of HB-EGF has previously been shown to be increased by $H$ pylori. ${ }^{6}$ All three gastric epithelial cells exposed to $H$ pylori strain 60190 showed a significant increase in HB-EGF gene expression $(\mathrm{p}<0.001$; figure $2 \mathrm{a})$, cell-associated protein expression as determined by immunofluorescence $(p<0.03$; figure $2 \mathrm{~b}$ and $\mathrm{c})$ and shedding as measured by an ELISA assay $(\mathrm{p}<0.01$; figure $2 \mathrm{~d}$ ). $H$ pylori strain Tx30a did not significantly upregulate HB-EGF gene expression (figure $2 \mathrm{a}$ ) and cell-associated expression (figure $2 \mathrm{~b}$ and $\mathrm{c}$ ) but did significantly upregulate HB-EGF shed- ding in AGS and ST16 cells $(\mathrm{p}<0.05)$, which was significantly lower than that seen with strain $60190(p<0.05)$ and had no effect on HB-EGF shedding in MGLVA1 cells (figure $2 \mathrm{~d}$ ).

MMP-7 upregulation in $\boldsymbol{H}$ pylori-infected cells and its role in regulation of soluble HB-EGF shedding and EMT

MMP-7 gene expression was significantly upregulated following exposure to $H$ pylori strain 60190 in all three gastric cell lines $(\mathrm{p}<0.001)$, but not following exposure to Tx30a (figure $3 \mathrm{a}$ ). MMP-7 protein expression, as determined by immunofluorescence, was upregulated by $H$ pylori strain 60190 but not Tx30a (figure $3 b$ and $c, p<0.001$ ) and co-localised with HB-EGF expression in H pylori 60190 infected cells (figure 3d).

MMP-7 siRNA, which significantly downregulated MMP-7 gene expression in all three gastric cell lines (figure $3 e, p<0.001$ ), also significantly reduced HB-EGF gene expression in all three cell lines (AGS, $\mathrm{p}<0.02$; MGLVA1, $\mathrm{p}<0.03$; ST16, $\mathrm{p}<0.02$; figure 3f) and MMP-7 neutralising antibody significantly downregulated $\mathrm{HB}-\mathrm{EGF}$ gene expression $(\mathrm{p}<0.03$ in all cell lines; figure $3 \mathrm{~g}$ ) and ectodomain shedding (ST16, $\mathrm{p}<0.03$; AGS, $\mathrm{p}<0.04$; MGLVA1, $\mathrm{p}<0.04$; figure $3 \mathrm{~h}$ ). Finally, MMP-7 siRNA was shown to downregulate $H$ pylori 60190 -induced EMT expression in all
Figure 2 Effect of co-culture of gastric epithelial cells with Helicobacter pylori strain 60190 on heparin-binding epidermal growth factor (HB-EGF) expression. (a) HB-EGF gene expression following co-culture of gastric epithelial cells with $H$ pylori pathogenic (60190) and non-pathogenic (Tx30a) strains; ${ }^{*} p<0.001$ ( $n=3$ replicates per condition and a representative graph is shown, error bars indicate 95\% confidence). (b) Cell-associated HB-EGF expression following co-culture of gastric epithelial cells with $H$ pylori pathogenic (60190) and non-pathogenic (Tx30a) strains. Cells were stained for HB-EGF (red fluorescence) and nuclei were counterstained with Hoechst (blue fluorescence); (i) AGS, (ii) AGS/H pylori 60190, (iii) AGS/H pylori Tx30a. Magnification $\times 20$. (c) HB-EGF protein expression level quantified by image analysis following co-culture of gastric epithelial cells with $H$ pylori pathogenic (60190) and non-pathogenic (Tx30a) strains; * significantly higher HB-EGF protein expression level compared with untreated control cells $(p<0.03)$.

(d) HB-EGF shedding level following co-culture of gastric epithelial cells with $H$ pylori pathogenic (60190) and non-pathogenic (Tx30a) strains; * significantly higher HB-EGF shedding level compared with untreated control cells (strain 60190 treated: $p<0.01$ in all cell lines; strain Tx30a treated: $p<0.05$ in AGS and ST16); \#significantly higher HB-EGF shedding level compared with Tx30a treated $(p<0.05$ in AGS and ST16). ( $n=3$ replicates per condition and a representative graph is shown, error bars indicate 95\% confidence).

a

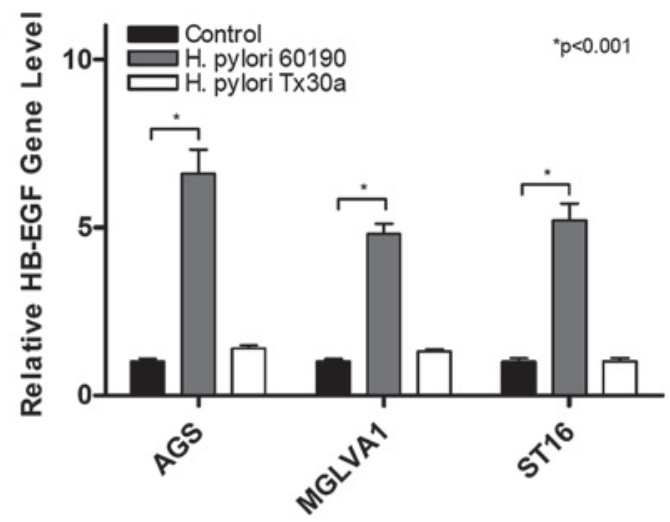

b

(i)

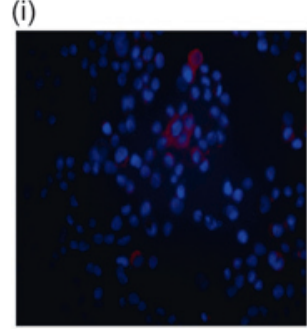

(ii)
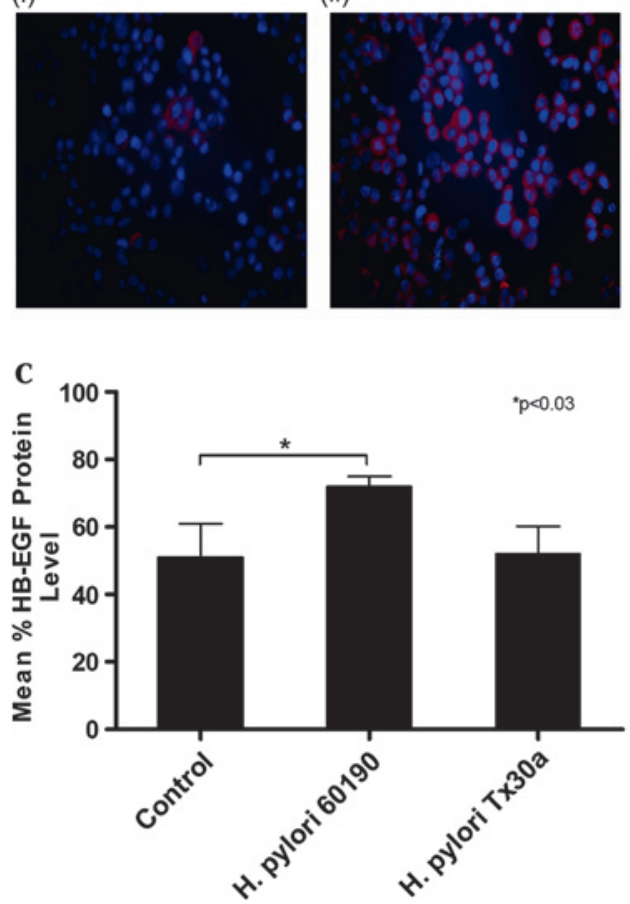

(iii)

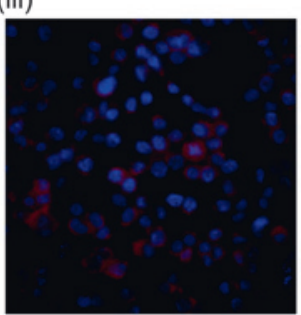

d

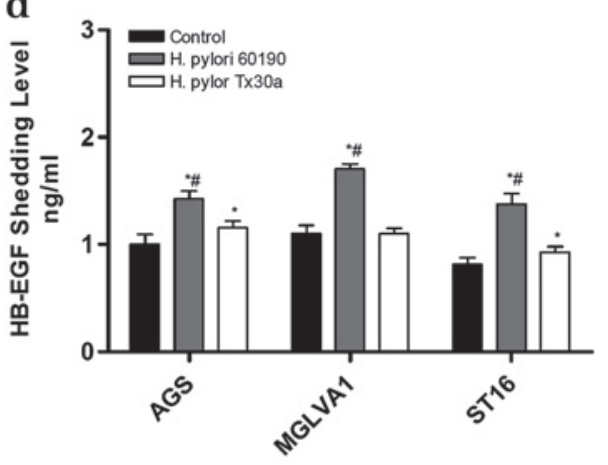


Figure 3 Effect of co-culture of gastric epithelial cells with Helicobacter pylori strain 60190 on matrix metalloproteinase 7 (MMP-7) expression. (a) MMP-7 gene expression following co-culture of gastric epithelial cells with $H$ pylori pathogenic (60190) and non-pathogenic (Tx30a) strains. ${ }^{*} \mathrm{p}<0.001$ ( $\mathrm{n}=3$ replicates per condition and a representative graph is shown, error bars indicate 95\% confidence).

(b) MMP-7 protein expression following co-culture of gastric epithelial cells with $H$ pylori pathogenic (60190) and nonpathogenic (Tx30a) strains. Cells were stained for MMP-7 (green fluorescence) and nuclei were counterstained with Hoechst (blue fluorescence). (i) AGS, (ii) AGS/H pylori 60190, (iii) AGS $H$ pylori $\mathrm{T} \times 30$ a. Magnification $\times 20$. (c) MMP-7 protein expression level quantified by image analysis following co-culture of gastric epithelial cells with $H$ pylori pathogenic (60190) and non-pathogenic (Tx30a) strains; ${ }^{*}$ significantly higher MMP-7 protein expression level compared with untreated control cells $(p<0.001)$. (d) MMP-7 and heparin-binding epidermal growth factor (HB-EGF) colocalisation: MMP-7 expression was stained by green fluorescence (i), HBEGF by red fluorescence (ii), nuclei were stained with Hoechst blue fluorescence (iii). Merged image shows co-localisation of MMP-7 and HB-EGF (iv). Magnification $\times 20$. (e) MMP-7 gene expression in MMP-7 siRNA treated gastric cell lines. MMP-7 siRNA significantly reduced MMP-7 mRNA expression in all three gastric cell lines tested. ${ }^{*} \mathrm{p}<0.001$ ( $\mathrm{n}=3$ replicates per condition and a representative graph is shown, error bars indicate $95 \%$ confidence). (f) HB-EGF gene expression in MMP-7 siRNA treated gastric cell lines exposed to $H$ pylori 60190. MMP-7 siRNA significantly reduced $\mathrm{HB}$-EGF shedding in all three gastric cell lines tested. ${ }^{*} \mathrm{p}<0.02$,
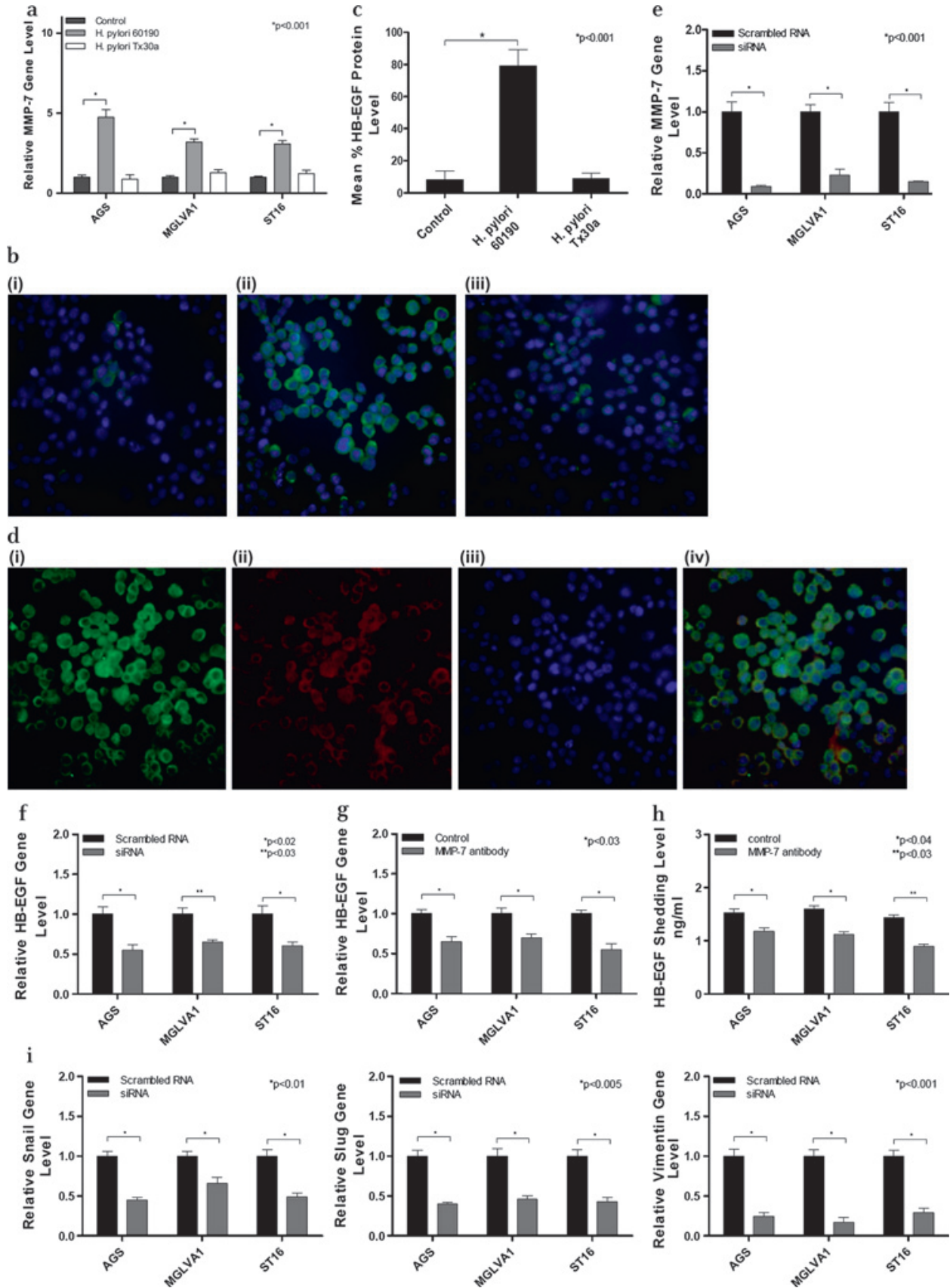

${ }^{* *} \mathrm{p}<0.03$ ( $\mathrm{n}=3$ replicates per condition and a representative graph is shown, error bars indicate $95 \%$ confidence). (g) HB-EGF gene expression in MMP-7 neutralising antibody treated gastric cell lines exposed to $H$ pylori $60190{ }^{*} \mathrm{p}<0.03(\mathrm{n}=3$ replicates per condition and a representative graph is shown, error bars indicate $95 \%$ confidence). (h) HB-EGF shedding levels in gastric cell lines exposed to $H$ pylori 60190 treated with an MMP-7 neutralising antibody. ${ }^{*} p<0.04,{ }^{* *} p<0.03$ ( $n=3$ replicates per condition and a representative graph is shown, error bars indicate $95 \%$ confidence). (i) EMT gene expression in MMP-7 siRNA treated gastric cell lines exposed to $H$ pylori strain 60190 : Snail, $p<0.01 ;$ Slug, $p<0.005$; vimentin, $p<0.001$ ( $n=3$ replicates per condition and a representative graph is shown, error bars indicate $95 \%$ confidence).

three gastric cell lines (Snail, $p<0.01$; Slug, $p<0.005$; vimentin, $\mathrm{p}<0.001$; figure $3 \mathrm{i}$ ).

\section{H pylori 60190 upregulates gastrin expression and gastrin siRNA inhibits $\boldsymbol{H}$ pylori-induced MMP-7 and EMT expression}

All three gastric cell lines showed significantly increased levels of gastrin expression following exposure to $H$ pylori strain 60190 $(p<0.001)$ whereas exposure to $H$ pylori strain Tx30a had no significant effect on gastrin gene expression (figure 4a). As both gastrin and MMP-7 expression were upregulated following
$H$ pylori infection, experiments were carried out to investigate whether the upregulation of MMP-7 following $H$ pylori infection was a result of the upregulation of gastrin gene expression. Gastrin gene expression was decreased $>90 \%$ in all three gastric epithelial cell lines using a gastrin siRNA (figure 4b, $\mathrm{p}<0.01$ ). This gastrin siRNA has previously been shown to significantly reduce $H$ pylori-induced $\mathrm{HB}$-EGF gene expression and shedding in gastric epithelial cell lines, and in our study it was also shown to significantly reduce upregulation of MMP-7 induced by $H$ pylori strain 60190 (figure 4c, p<0.02). ${ }^{6}$ However, ST16 cells showed 


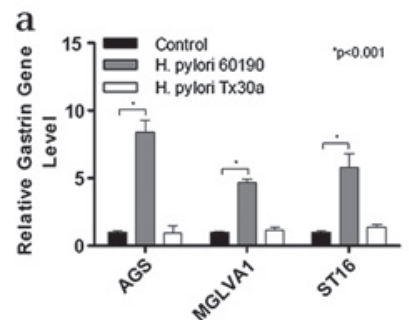

d AGS

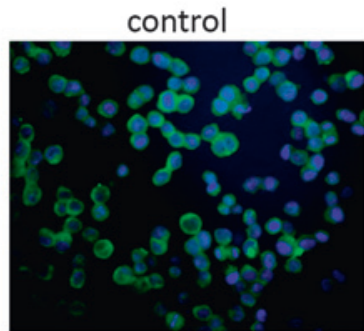

MGLVA1

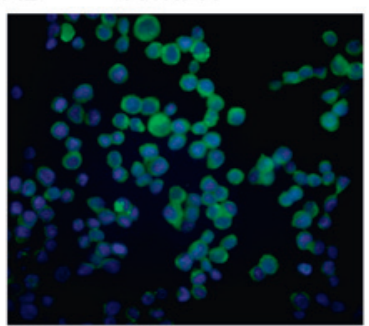

ST16

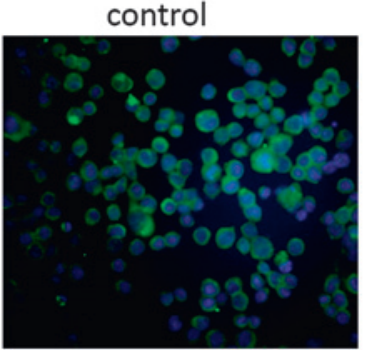

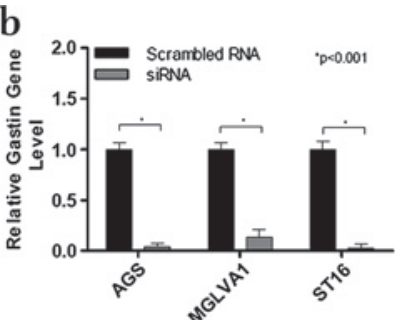

siRNA transfected

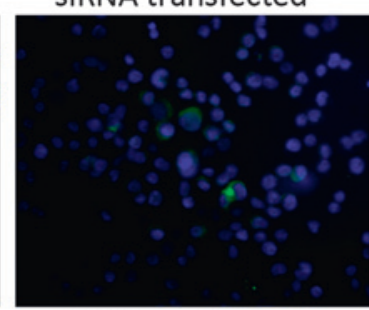

siRNA transfected

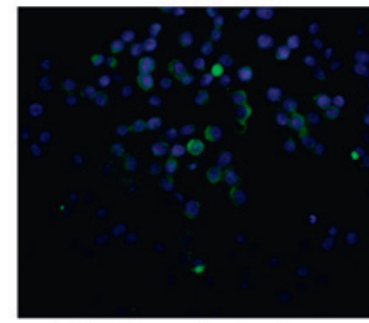

siRNA transfected

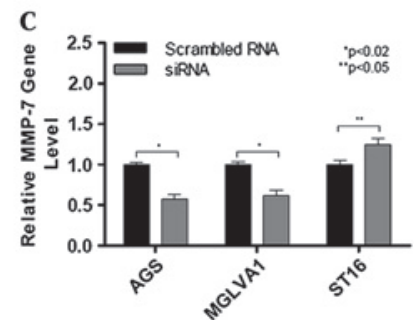

e

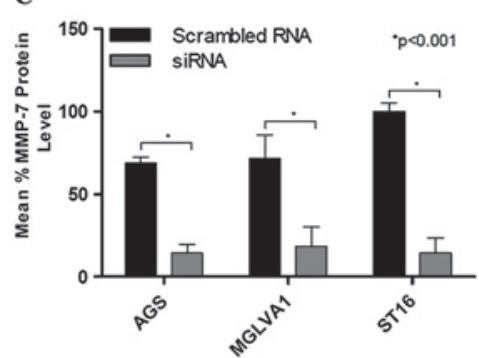

a small but significant increase in MMP-7 expression after treatment with gastrin siRNA (figure $4 \mathrm{c}, \mathrm{p}<0.05$ ).

Gastrin siRNA-transfected AGS and MGLVA1 cells showed reduced MMP-7 protein expression compared with control and, despite an increase in mRNA levels, the protein expression of
MMP-7 was also significantly reduced in gastrin siRNA-transfected ST16 cells compared with the mock-transfected control (figure $4 \mathrm{~d}$ and $\mathrm{e}$ ). In addition, gastrin siRNA was shown to significantly reduce $H$ pylori 60190-induced EMT gene expression of Snail and Slug and the mesenchymal cytoskeletal protein 
a (i)

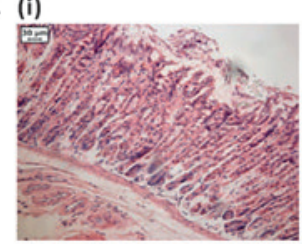

(ii)

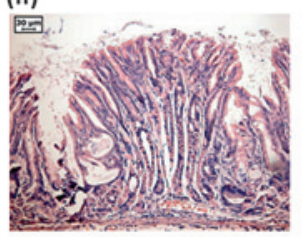

b

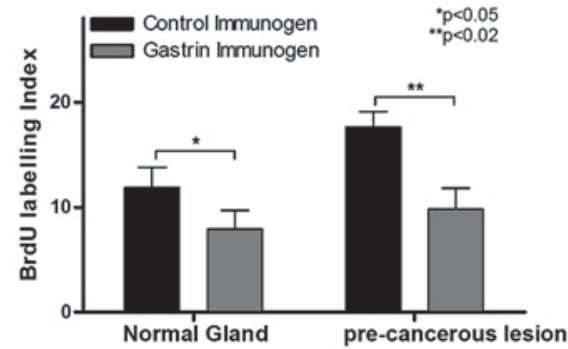

C MMP-7 expression

(i)

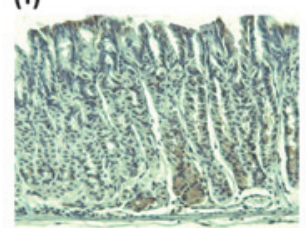

HB-EGF expression

(i)

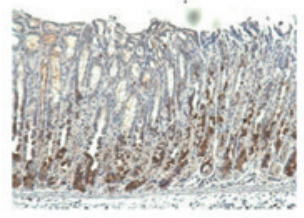

Slug Expression

(i)

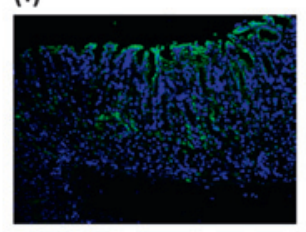

d

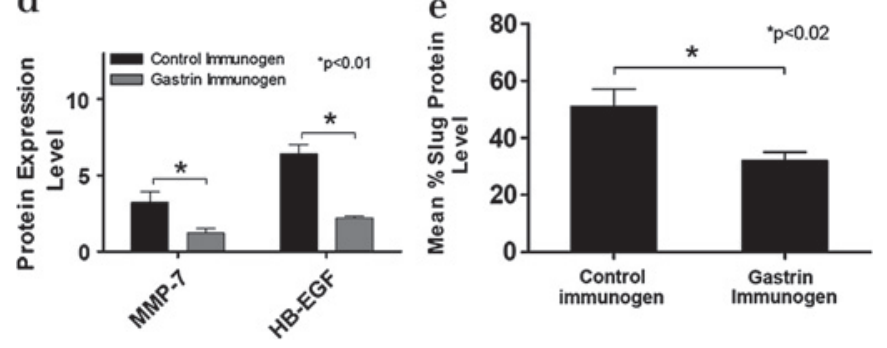

Figure 5 Effect of gastrin neutralisation on matrix metalloproteinase 7 (MMP-7), heparin-binding epidermal growth factor (HB-EGF) and epithelial:mesenchymal transition (EMT) expression in the INS-GAS transgenic mouse model of gastric carcinogenesis. (a) Haematoxylin and eosin stained INS-GAS samples treated with gastrin immunogen (i) or control immunogen (ii). Magnification $\times 20$. (b) Quantification of levels of proliferation in INS-GAS samples treated with gastrin immunogen or control immunogen. ${ }^{*} p<0.05,{ }^{* *} p<0.02$ (5 animals per group, error bars indicate SD). (c) MMP-7, HB-EGF and Slug expression in malignant lesions in the gastric mucosa of gastrin immunogen (i) and control vimentin in all three gastric cell lines (Snail, $\mathrm{p}<0.001$; Slug, $\mathrm{p}<0.01$; vimentin, $\mathrm{p}<0.01$; figure $4 \mathrm{f}$ ).

Proof of concept study: INS-GAS mouse in vivo carcinogenesis model

Finally, the involvement of the gastrin-induced EMT genes, MMP-7 and HB-EGF in carcinogenesis in the INS-GAS mouse stomach was investigated.

INS-GAS mice were treated with the gastrin immunogen G17DT to reduce serum gastrin levels, with controls treated with an inactive form of the immunogen DT. Thirty weeks following treatment, histological examination revealed that there were fewer abnormal/premalignant changes of the fundic mucosa in G17DT-immunised mice than in the DT control immunised INS-GAS mice (figure $5 \mathrm{a}$ ). In addition, BrdU staining showed that in both the normal glands and precancerous lesions, G17DT treatment resulted in significantly reduced proliferation (3.9\% in normal glands vs $7.6 \%$ in precancerous lesions) compared with the DT control group $(p<0.05$ and $p<0.02$, respectively, figure $5 b$ ). MMP-7, Slug and HB-EGF levels were significantly reduced by $66 \%, 64 \%$ and $36 \%$ respectively in the G17DT-treated group compared with the DT control group $(p<0.01$ for HB-EGF, $p<0.01$ for MMP-7, $p<0.02$ for Slug; figure $5 c$ and $d)$.

\section{DISCUSSION}

Using an in vitro $H$ pylori co-culture system, this study shows that a pathogenic $H$ pylori strain (60190) upregulated EMT in a series of human gastric cell lines while a non-pathogenic $H$ pylori strain (Tx30a) had much reduced effects. This effect of $H$ pylori on EMT markers may happen in a cagPaI-related manner. It was hypothesised that this could have been mediated partly via the known ability of $H$ pylori to upregulate HB-EGF, as the soluble form of the growth factor has been implicated in EMT and that HB-EGF ectodomain shedding was facilitated by MMP-7, another factor increased in $H$ pylori infections. ${ }^{6-8}{ }^{14-16}$ Finally, our investigations centred on the link with gastrin, which is known to be increased in the serum of $H$ pylori-infected patients and at the cellular level in $H$ pylori-infected cells. ${ }^{2} 27$ HB-EGF shedding from gastric epithelial cells was significantly increased following treatment with $H$ pylori strain 60190 while less change was detected in cells co-cultured with $H$ pylori strain Tx30a. It has been reported that the activation of the bacterial enzyme $\gamma$-glutamyltranspeptidase results in activation of PI3K and p38 kinase-dependent signal transduction pathways which then lead to increased HB-EGF expression. ${ }^{29}$ Work in our laboratory has shown that gastrin and the CCK-2 receptor are involved in the increased shedding and gene expression of HB-EGF following $H$ pylori infection. ${ }^{6}$

When gastric cell lines were exposed to $H$ pylori strain 60190, MMP-7 gene and protein expression were significantly

[continued]

immunogen (ii) treated mice. Samples were stained by immunohistochemistry for HB-EGF and MMP-7 (brown staining indicates positive expression) or by immunofluorescence for Slug (green fluorescence indicates positive expression). An area representative of staining in nine stomach sections is shown. The appearance of the other sections was similar. Magnification $\times 20$. (d) Protein expression levels of MMP-7, HB-EGF and Slug in the INS-GAS transgenic mouse model of gastric carcinogenesis. Staining was quantified by image analysis in both gastrin immunogen and control immunogen treated mice. MMP-7, $p<0.01$; HB-EGF, $p<0.01$; Slug, $p<0.02(n=9$, error bars indicate SD). 
upregulated. The same cell lines treated with $H$ pylori strain Tx30a showed no change in MMP-7 expression. The major difference between these two laboratory-adapted strains is the presence of an intact and functional cagPaI in strain 60190, and we speculate that the differences we observed in our studies are due to this locus. Strain 60190 also possesses a vacuolating form of VacA whereas strain Tx30a possesses a non-vacuolating form, but previous work has shown that MMP-7 upregulation by strain 60190 is not VacAdependent. The observed increase in MMP-7 expression is consistent with the literature in that gastric biopsy specimens from $H$ pylori positive patients expressed much higher levels of MMP-7 at both the gene and protein level. ${ }^{17} \mathrm{H}$ pylori infection in gastric epithelial cells activates Activator protein 1 (AP-1) and nuclear factor- $\kappa \mathrm{B}$, which may lead to MMP-7 overexpression. ${ }^{15} 3031$ It has also been shown that cagPaI positive $H$ pylori strains activated ERK $1 / 2$ and p38 and downstream MMP-7 expression. ${ }^{32}$

Inhibition of MMP-7 activity by either MMP-7 specific siRNA or a neutralising antibody significantly reduced the increased HB-EGF shedding by $H$ pylori strain 60190, indicating that increased HB-EGF shedding following $H$ pylori infection is at least partially a result of increased MMP-7 expression. In addition, MMP-7 siRNA significantly reduced $H$ pylori-induced EMT.

MMP-7 has long been recognised as an important player involved in HB-EGF shedding, and in the present study it was shown by immunofluorescence that MMP-7 and HB-EGF are expressed in a similar subcellular area. ${ }^{33} 34$ It has been reported that CD44 heparin sulphate proteoglycan recruits MMP-7 and pro-HB-EGF to form a complex on the surface of a tumour cell line, which provides a mechanism for the regulation of HB-EGF processing. ${ }^{33}$ It has also been reported that $H$ pylori increases HB-EGF shedding through upregulation of MMPs to trigger constitutive EGFR signal activation, which initiates neoplastic transformation of gastric epithelial cells. ${ }^{35}$

All three gastric cell lines showed significant upregulation of gastrin expression after exposure to the pathogenic $H$ pylori strain 60190, while no change in gastrin expression was observed in the same cell lines treated with the non-pathogenic $H$ pylori strain, as expected. ${ }^{6}$ Attachment of $H$ pylori to gastric epithelial cells induces chronic inflammatory responses which lead to increased expression of various cytokines in a cagPaI-dependent manner. ${ }^{36}{ }^{37}$ These include interleukin 8 and tumour necrosis factor $\alpha$, which have been reported to increase gastrin expression. H pylori also activates gastrin releasing peptide (through the type IV secretion system) and regulates the gastrin modulator somatostatin to increase gastrin expression. ${ }^{38} 39$

It has previously been confirmed that gastrin is involved in the upregulation of both MMP-7 and HB-EGF by H pylori. ${ }^{6} 1819$ Gastrin knock-down by siRNA significantly reduced MMP-7 protein expression in all three gastric cell lines, but MMP-7 gene expression was only downregulated in AGS and MGLVA1 cells. This may be due to post-transcriptional regulation of MMP-7-for example, through miRNA-mediated mechanisms - reducing levels of MMP-7 protein in spite of upregulation at the mRNA level in ST16 cells. A lack of concordance between MMP-7 mRNA and protein levels has previously been observed in the context of breast cancer. ${ }^{40}$ It has also been reported that the tissue inhibitors of metalloproteinase (TIMPs) are upregulated following $H$ pylori infection in a gastrin-associated manner. ${ }^{41}$ This indicates that gastrin may affect the MMP and TIMP balance following $H$ pylori infection. Gastrin has also been reported to upregulate MMP-7 through activation of NF- $\mathrm{B}$ via a protein kinase C-dependent pathway which involves I $\mathrm{B}$ kinase. ${ }^{42}$

In addition to soluble HB-EGF increasing EMT, MMP-7 overexpression is known to activate EMT signalling pathways to regulate myofibroblast function via MAPK and PI3K pathways. ${ }^{20}$ In addition, MMP-7, together with MMP-3, is involved in the initiation of EMT by cleavage of E-cadherin which leads to tumour cell migration from the primary tumour, a process leading to distant metastases. ${ }^{43}$

In the hypergastrinaemic transgenic mouse model of gastric carcinogenesis, both MMP-7 and HB-EGF have previously been shown to be upregulated. ${ }^{25}$ The expression of EMT within abdominal gastric lesions in this model was confirmed and reduction of gastrin expression using a gastrin immunogen reduced the EMT gene Slug, as well as both MMP-7 and HB-EGF. This in vivo study begins to confirm the link between HB-EGF, MMP-7 and EMT shown at the cellular level in the presence of $H$ pylori. In an oesophageal adenocarcinoma setting, a recent paper has also confirmed the involvement of Slug in adenocarcinoma progression which was linked to E-cadherin expression and increased levels of vimentin. ${ }^{44}$

In conclusion, $H$ pylori increases EMT in the gastric cancer setting partially mediated by a cooperative network involving MMP-7, HB-EGF and gastrin.

Funding Core.

Competing interests None.

Provenance and peer review Not commissioned; externally peer reviewed.

\section{REFERENCES}

1. Starzynska T, Malfertheiner P. Helicobacter and digestive malignancies. Helicobacter 2006;11(Suppl 1):32-5.

2. Watson SA, Grabowska AM, El-Zaatari M, et al. Gastrin-active participant or bystander in gastric carcinogenesis? Nat Rev Cancer 2006;6:936-46.

3. Censini S, Lange C, Xiang Z, et al. Cag, a pathogenicity island of Helicobacter pylori, encodes type I-specific and disease-associated virulence factors. Proc Natl Acad Sci U S A 1996;93:14648-53.

4. Huang J0, Zheng GF, Sumanac K, et al. Meta-analysis of the relationship between cagA seropositivity and gastric cancer [see comment]. Gastroenterology 2003; 125:1636-44

5. Schlessinger J, Schlessinger J. Common and distinct elements in cellular signaling via EGF and FGF receptors. Science 2004;306:1506-7.

6. Dickson JH, Grabowska A, El-Zaatari M, et al. Helicobacter pylori can induce heparin-binding epidermal growth factor expression via gastrin and its receptor Cancer Res 2006;66:7524-31.

7. Smith JP, Pozzi A, Dhawan P, et al. Soluble HB-EGF induces epithelial-tomesenchymal transition in inner medullary collecting duct cells by upregulating Snail2. Am J Physiol Renal Physiol 2009;296:F957-65.

8. Yagi H, Yotsumoto F, Miyamoto S. Heparin-binding epidermal growth factor-like growth factor promotes transcoelomic metastasis in ovarian cancer through epithelial-mesenchymal transition. Mol Cancer Ther 2008; 7:3441-51.

9. Bagnoli F, Buti L, Tompkins L, et al. Helicobacter pylori CagA induces a transition from polarized to invasive phenotypes in MDCK cells. Proc Natl Acad Sci U S A 2005; 102:16339-44.

10. Schafer B, Gschwind A, Ullrich A, et al. Multiple G-protein-coupled receptor signals converge on the epidermal growth factor receptor to promote migration and invasion. Oncogene 2004;23:991-9.

11. Mammoto T, Higashiyama S, Mukai M, et al. Infiltration anesthetic lidocaine inhibits cancer cell invasion by modulating ectodomain shedding of heparin-binding epiderma growth factor-like growth factor (HB-EGF). J Cell Physiol 2002;192:351-8.

12. Wang $\mathbf{F}$, Sloss $C$, Zhang $X$, et al. Membrane-bound heparin-binding epidermal growth factor like growth factor regulates $\mathrm{E}$-cadherin expression in pancreatic carcinoma cells. Cancer Res 2007:67:8486-93.

13. Goishi K, Higashiyama S, Klagsbrun $\mathbf{M}$, et al. Phorbol ester induces the rapid processing of cell surface heparin-binding EGF-like growth factor: conversion from juxtacrine to paracrine growth factor activity. Mol Biol Cell 1995;6:967-80.

14. Kato N, Shimmura S, Kawakita T, et al. Beta-catenin activation and epithelialmesenchymal transition in the pathogenesis of pterygium. Invest Ophthalmol Vis Sci 2007; 48:1511-17.

15. Wroblewski LE, Noble PJ, Pagliocca A, et al. Stimulation of MMP-7 (matrilysin) by Helicobacter pylori in human gastric epithelial cells: role in epithelial cell migration. J Cell Sci 2003;116:3017-26.

16. McCaig C, Duval C, Hemers E, et al. The role of matrix metalloproteinase-7 in redefining the gastric microenvironment in response to Helicobacter pylori. Gastroenterology 2006;130:1754-63.

17. Bebb JR, Letley DP, Thomas RJ, et al. Helicobacter pylori upregulates matrilysin (MMP-7) in epithelial cells in vivo and in vitro in a Cag dependent manner. Gut 2003;52:1408-13. 
18. Kermorgant S, Lehy T. Glycine-extended gastrin promotes the invasiveness of human colon cancer cells. Biochem Biophys Res Commun 2001;285:136-41.

19. Wroblewski LE, Pritchard DM, Carter S, et al. Gastrin-stimulated gastric epithelial cell invasion: the role and mechanism of increased matrix metalloproteinase 9 expression. Biochem J 2002;365:873-9.

20. Varro A, Kenny S, Hemers $E$, et al. Increased gastric expression of MMP-7 in hypergastrinemia and significance for epithelial-mesenchymal signaling. Am J Physiol Gastrointest Liver Physiol 2007;292:G1133-40.

21. Watson SA, Durrant LG, Wencyk PM, et al. Intracellular gastrin in human gastrointestinal tumor cells. J Natl Cancer Inst 1991;83:866-71.

22. Harris JC, Clarke PA, Awan A, et al. An antiapoptotic role for gastrin and the gastrin/CCK-2 receptor in Barrett's esophagus. Cancer Res 2004;64:1915-19.

23. Grabowska AM, Hughes J, Watson SA. Use of interfering RNA to investigate the role of endogenous gastrin in the survival of gastrointestinal cancer cells. $\mathrm{Br} \mathrm{J}$ Cancer 2007:96:464-73.

24. Livak KJ, Schmittgen TD. Analysis of relative gene expression data using real-time quantitative PCR and the 2(-Delta Delta C(T)) method. Methods 2001:25:402-8.

25. Wang TC, Dangler CA, Chen D, et al. Synergistic interaction between hypergastrinemia and Helicobacter infection in a mouse model of gastric cancer. Gastroenterology 2000;118:36-47.

26. Watson SA, Michaeli D, Grimes S, et al. Gastrimmune raises antibodies that neutralize amidated and glycine-extended gastrin-17 and inhibit the growth of colon cancer. Cancer Res 1996:56:880-5.

27. Smith JT, Pounder RE, Nwokolo CU, et al. Inappropriate hypergastrinaemia in asymptomatic healthy subjects infected with Helicobacter pylori. Gut 1990;31:522-5.

28. Levi S, Beardshall K, Swift I, et al. Antral Helicobacter pylori, hypergastrinaemia, and duodenal ulcers: effect of eradicating the organism. BMJ 1989;299:1504-5.

29. Busiello I, Acquaviva R, Di Popolo A, et al. Helicobacter pylori gammaglutamyltranspeptidase upregulates COX-2 and EGF-related peptide expression in human gastric cells. Cell Microbiol 2004;6:255-67.

30. Meyer-ter-Vehn T, Covacci A, Kist M, et al. Helicobacter pylori activates mitogenactivated protein kinase cascades and induces expression of the proto-oncogenes Cfos and c-jun. J Biol Chem 2000;275:16064-72.

31. Naumann M, Wessler S, Bartsch C, et al. Activation of activator protein 1 and stress response kinases in epithelial cells colonized by Helicobacter pylori encoding the cag pathogenicity island. J Biol Chem 1999:274:31655-62.

32. Crawford HC, Krishna US, Israel DA, et al. Helicobacter pylori strain-selective induction of matrix metalloproteinase-7 in vitro and within gastric mucosa. Gastroenterology 2003:125:1125-36.
33. Yu WH, Woessner JF Jr, McNeish JD, et al. CD44 anchors the assembly of matrilysin/MMP-7 with heparin-binding epidermal growth factor precursor and ErbB4 and regulates female reproductive organ remodeling. Genes Dev 2002;16:307-23.

34. Umata T, Hirata M, Takahashi T, et al. A dual signaling cascade that regulates the ectodomain shedding of heparin-binding epidermal growth factor-like growth factor. J Biol Chem 2001:276:30475-82.

35. Wallasch C, Crabtree JE, Bevec D, et al. Helicobacter pylori-stimulated EGF recepto transactivation requires metalloprotease cleavage of HB-EGF. Biochem Biophys Res Commun 2002;295:695-701.

36. Keates S, Sougioultzis S, Keates AC, et al. cag + Helicobacter pylori induce transactivation of the epidermal growth factor receptor in AGS gastric epithelial cells J Biol Chem 2001;276:48127-34.

37. Brandt S, Kwok T, Hartig R, et al. NF-kappaB activation and potentiation of proinflammatory responses by the Helicobacter pylori CagA protein. Proc Natl Acad Sci U S A 2005:102:9300-5.

38. Rieder G, Merchant JL, Haas R. Helicobacter pylori cag-type IV secretion system facilitates corpus colonization to induce precancerous conditions in Mongolian gerbils [see comment]. Gastroenterology 2005;128:1229-42.

39. Brzozowski T, Konturek PC, Mierzwa M, et al. Effect of probiotics and triple eradication therapy on the cyclooxygenase (COX)-2 expression, apoptosis, and functional gastric mucosal impairment in Helicobacter pylori-infected Mongolian gerbils. Helicobacter 2006;11:10-20.

40. Kohrmann A, Kammerer U, Kapp M, et al. Expression of matrix metalloproteinases (MMPs) in primary human breast cancer and breast cancer cell lines: new findings and review of the literature. BMC Cancer 2009;9:188.

41. Bodger K, Ahmed S, Pazmany L, et al. Altered gastric corpus expression of tissue inhibitors of metalloproteinases in human and murine Helicobacter infection. J Clin Pathol 2008:61:72-8

42. Ogasa M, Miyazaki Y, Hiraoka $S$, et al. Gastrin activates nuclear factor kappaB (NFkappaB) through a protein kinase $\mathrm{C}$ dependent pathway involving NFkappaB inducing kinase, inhibitor kappaB (lkappaB) kinase, and tumour necrosis factor receptor associated factor 6 (TRAF6) in MKN-28 cells transfected with gastrin receptor. Gut 2003:52:813-19.

43. Huguenin M, Müller EJ, Trachsel-Rösmann S, et al. The metalloprotease meprinbeta processes E-cadherin and weakens intercellular adhesion. PLoS One 2008; 3:e2153.

44. Jethwa $\mathbf{P}$, Naqvi $\mathrm{M}$, Hardy RG, et al. Overexpression of Slug is associated with malignant progression of esophageal adenocarcinoma. World J Gastroenterol 2008; 14:1044-52. 